\title{
METALLICITY, STRUCTURE AND KINEMATICS OF THE MILKY WAY'S BULGE
}

\author{
DANTE MINNITI \\ European Southern Observatory \\ Karl-Schwarzschild-Str. 2 \\ D-85748 Garching bei München \\ Germany
}

\section{Introduction}

One of the unsolved problems of the Milky Way is the interplay between the different components (disk, bulge and halo) in the inner regions, where they may all reach their maximum density. As a first approach to attack this problem, we summarize the results of a photometric and spectroscopic study of the Galactic bulge based on $\sim 700 \mathrm{~K}$ and $\mathrm{M}$ giants in 3 fields (Minniti 1993a). Table 1 lists the position of the fields, their projected Galactocentric distance, number of stars with spectra at present, and estimated disk contamination.

TABLE 1. Caption text

\begin{tabular}{lcccc}
\hline Field & $\mathrm{l}, \mathrm{b}$ & $\mathrm{R}(\mathrm{kpc})$ & $\mathrm{N}$ & $N_{\text {disk }} / N_{\text {tot }}$ \\
\hline F588 & 8,7 & 1.5 & 300 & $5 \%$ \\
M22 & $10,-8$ & 1.7 & 100 & $6 \%$ \\
F588 & 12,3 & 1.7 & 300 & $40 \%$ \\
\hline
\end{tabular}

\section{Metallicity of the Bulge}

We determine metallicities for a large number of $K$ giants in these 3 fields based on medium resolution spectra. These abundances are derived by mea- 


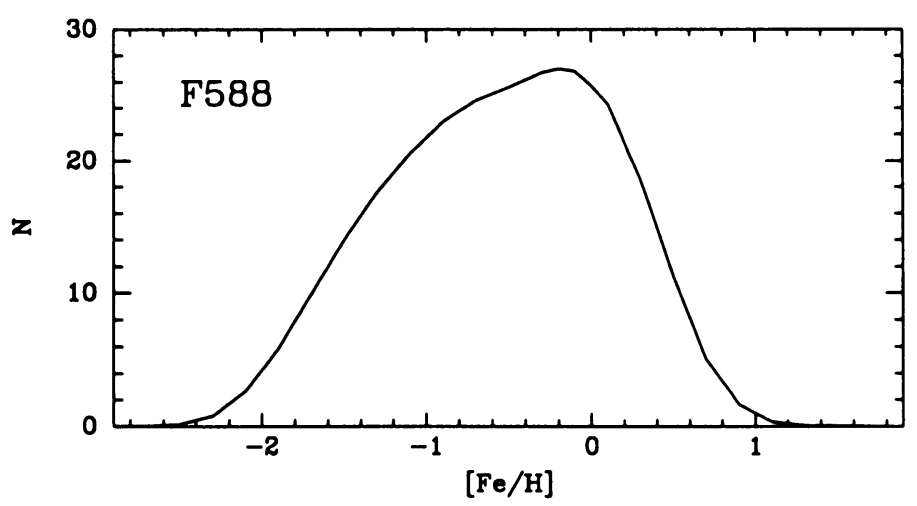

Figure 1. "True" metallicity distribution in field F588 obtained by Lucy deconvolution using the estimated errors, normalized to the total number of stars observed.

suring the strength of spectral indices, calibrated with a grid of giants observed in clusters with well-known abundances (Minniti et al. 1992). Our calibration is based on the globular cluster metallicity scale of Armandroff (1989). The mean metallicities for the fields with small disk contamination (M22 and F588) is between $[\mathrm{Fe} / \mathrm{H}]=-0.5$ and -0.6 . McWilliam \& Rich (1994) find a higher mean metallicity $([\mathrm{Fe} / \mathrm{H}]=-0.25)$ in Baade's Window, at $0.5 \mathrm{kpc}$ from the Galactic center.

From these data we conclude that: 1) There is a metallicity gradient within the inner $2 \mathrm{kpc}$ of the Galaxy, confirming previous photometric determinations (e.g. Terndrup 1988); 2) At any given distance from the Galactic center there is a large spread in metal abundances (Figure 1); and 3) The bulge is not super metal-rich as thought before. In particular, if the disk metallicity gradient continues or flattens out towards the Galactic center, the bulge itself would be more metal poor than the inner disk.

\section{Structure of the Bulge}

For the sample in the M22 field, we not only have abundances and optical photometry, but also proper motions measured by Cudworth (1986) and IR photometry. From these data we can estimate distances for the giants in this field if we assume ages for them. As a first guess, we adopt a mean age of $15 \mathrm{Gyr}$, for stars of all metallicities. The distribution of distances measured for the metal poor and metal rich giants is different (Figure 2a). Even though these distances can be uncertain, it is clear that: 1) the metal rich giants are closer in the mean than the metal poor giants, and 2) the metal rich giants have a more peaked distance distribution than the metal poor giants.

We compare the observed distributions with the ones predicted by the 

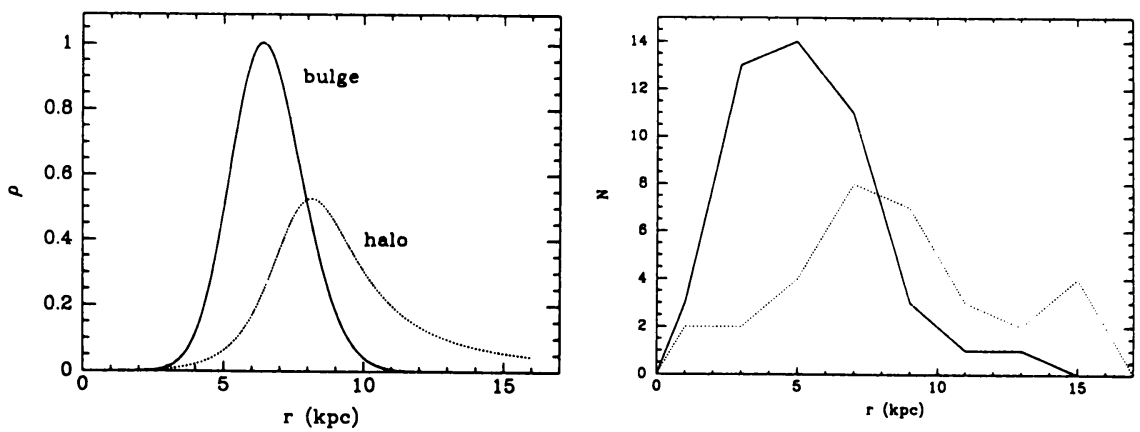

Figure 2. Left) Expected density distribution along the line of sight in the M22 field for both the halo (dotted line) and bulge (solid line), from the models described in the text. These densities have not been convolved with the error bars. Right) Observed distribution of stars as function of distance for the metal rich $([\mathrm{Fe} / \mathrm{H}] \geq-1$, solid line) and metal poor $([\mathrm{Fe} / \mathrm{H}] \leq-1$, dotted line) giants in the M22 field.

best fitting bar models for the bulge data from the DIRBE experiment on board the COBE satellite (Dwek et al. 1994), and by an axisymmetric halo with power law density distribution $\rho_{h} \propto r^{-3.2}$ (Figure 2b). The two observational results can be explained if there is a triaxial bulge consisting of stars with $[\mathrm{Fe} / \mathrm{H}] \geq-1$, and a halo that is more axisymmetric. This would be the first indication that the inner halo is not barred as the bulge. We also argue that in order to match the observed mean distance of bulge giants to models of the bar, the bulge must be significantly younger than the halo.

\section{Kinematics of the Bulge}

We also measured radial velocities with an accuracy of $\sim 10 \mathrm{~km} \mathrm{~s}^{-1}$ for all $\mathrm{K}$ and $\mathrm{M}$ stars. We will examine the kinematics of stars again in two groups: stars more metal-rich than $[\mathrm{Fe} / \mathrm{H}]=-1.0$, and metal-poor stars with $[\mathrm{Fe} / \mathrm{H}] \leq-1.0$. This division is chosen arbitrarily to reflect the transition between true halo stars and disk stars in the neighborhood of the Sun (e.g. Carney et al. 1989), and using the fact that there is no metallicity gradient in the halo (e.g. Zinn 1985).

For any given field there is a marked trend of kinematics with metallicity, in the sense that the metal-poor population has a higher velocity dispersion and a lower rotational velocity than the metal-rich population. An example of this is given in Figure 3. subsequently, we then look at the radial dependences of kinematics (Figure 4). It is found that the $\mathrm{K}$ giants with $[\mathrm{Fe} / \mathrm{H}] \leq-1$ have halo-like kinematics, with no significant rotation and $\sigma \sim 120 \mathrm{~km} \mathrm{~s}^{-1}$ independent of Galactocentric distance. The velocity dispersion of the giants with $[\mathrm{Fe} / \mathrm{H}] \geq-1$ decreases with increasing 

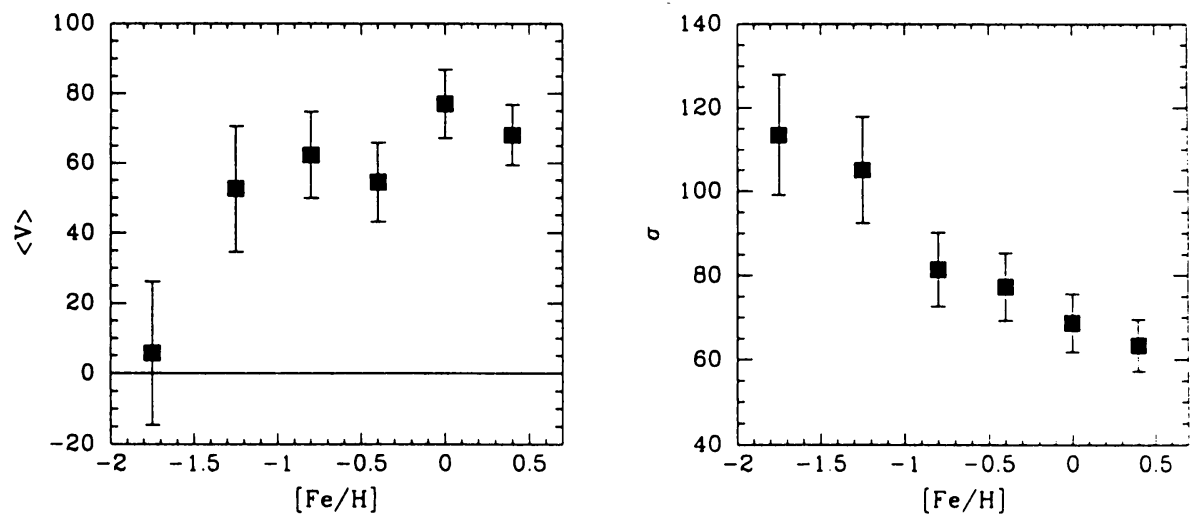

Figure 3. Mean Galactocentric velocities $V$, and velocity dispersions $\sigma$ vs metallicity for $\mathrm{K}$ giants in the $\mathrm{F} 588$ bulge field, located at $1, b=(8,7)$, at projected distance of 1.5 kpc from the Galactic center. Each bin includes 30 to 55 stars.

Galactocentric distance, and this population is rotating with $V \sim 9 \mathrm{~km} \mathrm{~s}^{-1}$ degree ${ }^{-1}$.

The velocity dispersions and rotational velocities of the metal-poor stars observed in bulge fields have similar values to those of the nearby halo stars, to those of the halo globulars and to those of the bulge RR Lyraes. On the basis of this comparison, it is then plausible to conclude that the metalpoor stars in this study, and the RR Lyraes in Baade's Window, are the extension of the halo population within $3 \mathrm{kpc}$. We would then conclude that the halo is isothermal all the way to the center.

The metal-rich population has substantial rotation, and we identify these stars with the bulge. The PN, Miras and SiO masers are kinematic tracers of the bulge and not the halo. Disk contamination would have a negligible effect in fields F588 and M22. Paczynski et al. (1994) model the number density of disk stars as constant up to some distance, and vanishing beyond that distance, as if the disk were hollow in the inner few kiloparsecs.

\section{The Formation of the Bulge and Halo}

Figure 5 shows the location of the halo and the bulge in a classical $\mathrm{V} / \sigma$ $v s \epsilon$ diagram (Binney 1978), where $\mathrm{V}$ is the peak rotation, $\sigma$ is the central velocity dispersion, and $\epsilon$ is the flattening ratio. This figure suggests that somehow during the formation of the Galaxy there was a change from an extended, pressure supported halo, to a flattened, more concentrated and rapidly rotating bulge. This leads to the following formation scenario: Only a small fraction of the gas in the proto-halo formed stars (e.g. Hartwick 1976). The rest of the gas was lost from the halo, sinking deep into the 

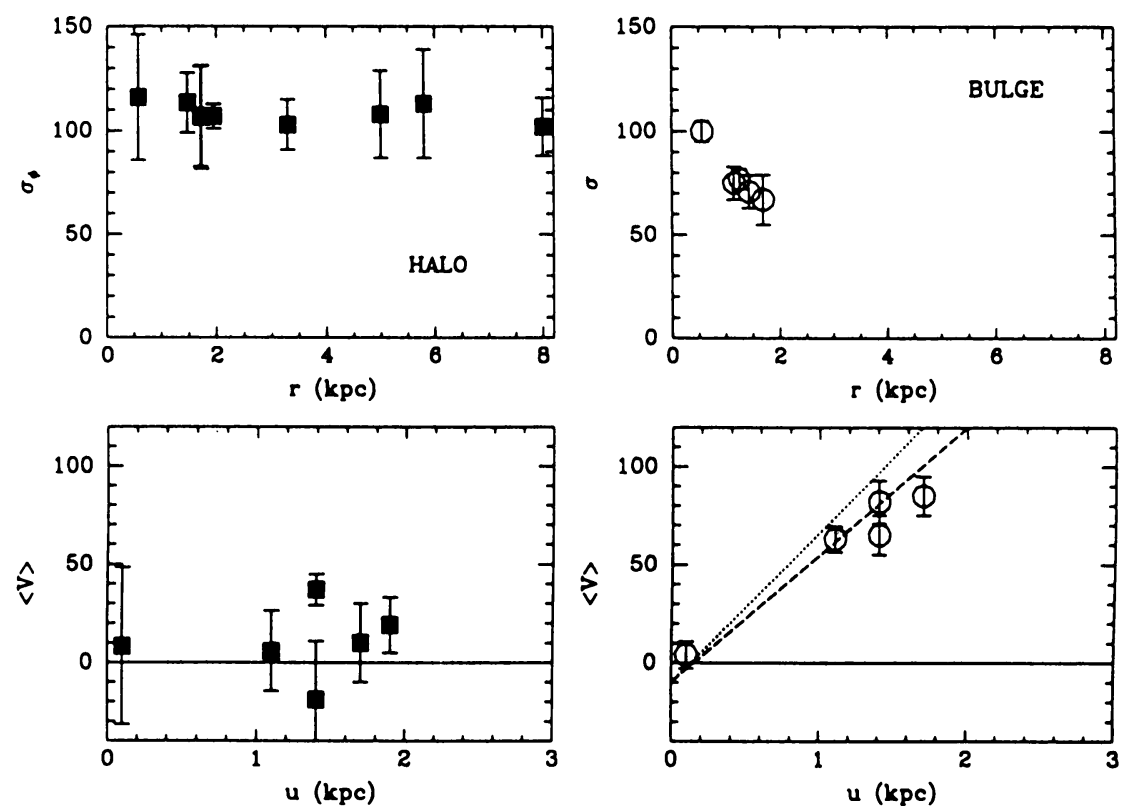

Figure 4. Run of the mean line of sight velocity $V$ vs Galactocentric distance projected in the plane $u$ and velocity dispersions $\sigma$ vs Galactocentric distance for $\mathrm{K}$ giants with $[\mathrm{Fe} / \mathrm{H}] \leq-1.0$ (left panels), and with $[\mathrm{Fe} / \mathrm{H}] \geq-1.0$ (right panels) in different fields towards the Galactic bulge. The dashed line in the lower right panel shows the mean rotation of planetary nebulae from Kinman et al. (1990), and the dotted line shows the mean rotation of bulge Mira variables (Menzies 1990), and of bulge SiO masers (Nakada et al. 1993).

potential, due to energy loss by radiation and cloud-cloud collisions, but conserving angular momentum. The enriched gas collapsed towards the central parts of the Galaxy, due to its low angular momentum, forming bulge stars (e.g. Carney et al. 1990, see also Wyse \& Gilmore 1992). In this model the bulge would have formed after the old halo stars (i.e. after most of the metal-poor RR Lyraes and globular clusters with blue horizontal branches).

The predictions from this scenario (and those from other models) can be tested with more data. In the nearby future we expect significant progress in the following areas: 1) Large number of proper motions in the bulge will lead to more refined dynamical models (e.g. Minniti 1993b, Zhao et al. 1994). 2) Accurate measurements of detailed chemical abundances for large numbers of bulge giants will lead to more refined models of chemical evolution (e.g. Matteucci \& Brocatto 1989). 3) Accurate ages obtained from observations with the refurbished HST combined with the metallicity information will lead to a better understanding of the chronology of bulge formation (e.g. Holtzmann et al. 1993). 


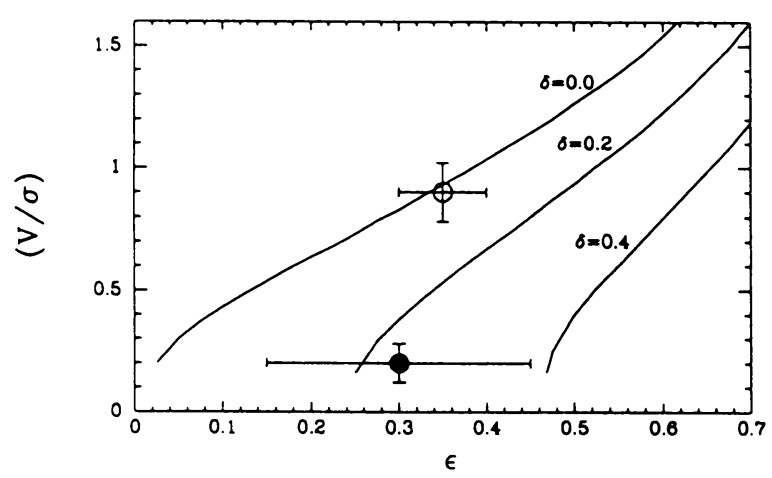

Figure 5. Relationship between rotation parameter $\mathrm{V} / \sigma$ and ellipticity $\epsilon$ for coaxial oblate spheroids with different anisotropy $\delta$ (solid lines), reproduced from Binney \& Tremaine (1987, their figure 4-5 on p. 217). We have plotted the location of the Galactic bulge (open circle) and halo (filled circle). The bulge ellipticity is taken from Kent et al. (1991), and the halo ellipticity from the reviews by Bahcall (1986) and Freeman (1987). The $1 \sigma$ error bars shown are conservative.

\section{Acknowledgements}

I am very grateful to $M$. Rich for reading my contribution during the meeting. I also thank L. Kaper for carefully reading this manuscript.

\section{References}

Armandroff, T. 1989 AJ 97, 375.

Bahcall, J. N. 1986 ARA\&A 24, 577.

Binney, J. 1978 MNRAS 183, 501.

Binney, J., \& Tremaine, S. 1987 "Galactic Dynamics" (Princeton Univ.), p. 217

Carney, B. W., Latham, D. W., \& Laird, J. B. 1990 AJ 99, 752.

Cudworth, K. M. 1986 AJ 92, 348.

Dwek, E. et al. $1994 A p J$, submitted.

Eggen, O. J., Lynden-Bell, D., and Sandage, A. 1962 ApJ 136, 748.

Hartwick, F. D. A. 1976 ApJ 209, 418.

Kent, S. M., Dame, T. M., \& Fazio, G. 1991 ApJ 378, 496.

Kinman, T. D., Feast, M. W., and Lasker, B. M. 1988 AJ 95, 804.

McWilliam, A., \& Rich, R. M. 1994 ApJS 91, 749.

Menzies, W. J. 1990 in "Bulges of Galaxies", eds. B. J. Jarvis \& D. Terndrup, p. 111.

Matteucci, F, \& Brocatto, E. 1990 ApJ 365, 359.

Minniti, D., White, S.D.M., Olszewski, E., and Hill, J.H. 1992 ApJ 393, L47.

Minniti, D. 1993a PhD Thesis, Steward Observatory, Univ. of Arizona.

Minniti, D. 1993b in "Galactic Bulges", B. J. Jarvis \& D. Terndrup, p. 315.

Nakada, Y., et al. 1993 PASJ 45, 179.

Paczynski, B., et al. 1994 AJ 107, 2060.

Rich, R. M. $1988 A J$ 95, 828.

Terndrup, D. M. $1988 \quad A J$ 96, 884.

Wyse, R. F. G., and Gilmore, G. $1992 \quad A J$ 104, 144.

Zhao, H.-S., Spergel, D., \& Rich, R. M. $1994 A J$, in press.

Zinn, R. 1985 ApJ 293, 424. 